\title{
Habilidades matemáticas básicas en alumnos de 3을 de Infantil: detección temprana de dificultades de aprendizaje y orientaciones para la intervención
}

\author{
Habilidades matemáticas básicas em crianças de 5 anos: \\ detecção precoce de dificuldades de aprendizagem e \\ orientações para a intervenção
}

\author{
Maria Cristina Núñez del Río ${ }^{[a]}$, Maria Isabel Pascual Gómez ${ }^{[b]}$ \\ [a] Profesora titular interina, Universidad Complutense de Madrid, Madrid, España, e-mail: \\ mcnunez@edu.ucm.es \\ [b] Profesora ayudante, Doctora, Universidad de Alcalá de Henares - Madrid, Madrid. España, e-mail: \\ isabel.pascualg@uah.es
}

\section{Resumen}

El presente trabajo aborda el estudio de las habilidades matemáticas básicas de alumnos de Educación Infantil, con el objetivo de detectar aquellos que manifiestan dificultades concretas en su aprendizaje y así poder orientar la intervención para su mejora. Se evaluó alumnos de 3a Infantil $(N=55)$ con el test TEMA-3 y la batería BADyG. El análisis de datos mediante ANOVA simple reveló la igualdad de las medias entre grupos; las diferencias de 
proporciones de logro en cada elemento de la prueba, a través de "Chi Cuadrado", mostraron las fortalezas y debilidades de los alumnos evaluados. Finalmente, el análisis de los resultados individuales permitió señalar aquellos que precisan especial atención. Se analizan las implicaciones educativas que se derivan de estos resultados.

Palabras-clave: Desarrollo matemático temprano. Competencia matemática temprana. Habilidades numéricas. Matemáticas en preescolar. Educación infantil.

\section{Resumo}

O presente trabalho aborda o estudo das habilidades matemáticas básicas de alunos de educação infantil, com o objetivo de detectar aqueles que manifestam dificuldades concretas na sua aprendizagem e assim poder orientar a intervenção para a sua melhora. Avaliaram-se alunos da 3a Infantil $(N=55)$ com o teste TEMA-3 e a bateria BADyG. A análise de dados mediante ANOVA simples revelou a igualdade das médias entre grupos; as diferenças de proporções de lucro em cada elemento da prova, por meio de "Qui-quadrado", mostraram as fortalezas e debilidades dos alunos avaliados. Finalmente, a análise dos resultados individuais permitiu assinalar aqueles que precisam especial atenção. Analisamse os envolvimentos educativos que se derivam desses resultados.

Palavras-chave: Desenvolvimento matemático precoce. Concorrência matemática precoce. Habilidades numéricas. Matemáticas em pré-escolar. Educação infantil.

\section{Introducción}

Conocer y entender las habilidades que manifiestan los alumnos al afrontar las actividades de aprendizaje que se les propone debe ser el punto inicial de toda intervención (BAROODY, 2000, 2001). Como ya recogía el National Council of Teacher of Mathematics (NCTM, 2000) y recientemente afirman Gil y Vicent (2009), sin duda, la etapa de Educación Infantil resulta un momento evolutivo clave para estimular el sentido numérico y animar el desarrollo aritmético en los niños, dada su fuerte 
capacidad predictora con relación al rendimiento académico posterior (KILDAY; KINZIE, 2009).

Durante las tres últimas décadas se ha dado un gran impulso al estudio del desarrollo del pensamiento matemático infantil, y los resultados de las investigaciones sugieren que los niños preescolares, en los inicios de su proceso de escolarización, ya disponen de ciertos conocimientos y destrezas en el ámbito de la aritmética informal, que deberán transformar en conocimientos y destrezas formales para comprender la matemática del mundo cotidiano (entre otros, BAROODY, 1988; BAROODY; COSLICK, 1998; BAROODY; DOWKER, 2003; BRISSIAUD, 1993; KAMII, 1985, 1992, 1995; NUNES; BRYANT, 1996). Diversos estudios de investigación han permitido establecer el largo recorrido que el niño realiza en su andadura inicial en el aprendizaje aritmético. Sin lugar a dudas, este desarrollo se inicia con la adquisición y elaboración de la secuencia numérica. Fuson, Richards y Briars (1982) describen el proceso completo, señalando las habilidades que los niños van teniendo disponibles en cada nivel de secuencia que van alcanzando. Pero el dominio de la secuencia numérica no es la meta final, sino una adquisición previa al logro de la comprensión del sistema numérico decimal, uno de los aprendizajes básicos de los primeros años de escolaridad. Resnick (1983) propone un modelo con claras repercusiones didácticas. Estos logros, más adelante, permitirán utilizar el cálculo de forma segura y apropiada. La observación sistemática de las actuaciones de los alumnos en las aulas de educación infantil, entre los 3 y los 5 años, ponen de manifiesto grandes variaciones en el ritmo de evolución de cada alumno, a lo largo de este proceso (NCTM, 2000; DOWKER, 2008). Tales diferencias justifican la necesidad de evaluación e intervención en esta etapa crucial del desarrollo, puesto que los alumnos aprenden a lo largo de ella los rudimentos del conocimiento aritmético, y por tanto, resultaría de vital importancia poder detectar, de forma temprana, las dificultades que pudieran surgir, de manera que se pueda promover su tratamiento y corrección (NCTM, 2000; BAROODY; BENSON, 2001).

La limitada información que, de forma estandarizada y formal, se podía obtener hasta ahora sobre las habilidades numéricas de los infantes 
ha condicionado la evaluación temprana que se podía realizar. En general sólo se disponía del análisis de los resultados obtenidos en escalas específicas de baterías globales (como el WIPPSI, McCARTHY, BADyG, EVALUA, BAPAE), o pruebas elaboradas particularmente para valorar aspectos concretos, como la aplicación de los principios de conteo o el nivel de habilidad en secuencia numérica.

Afortunadamente, en los últimos años, han surgido instrumentos y procedimientos variados que específicamente valoran la competencia matemática básica, con el claro objetivo de conocer y determinar las dificultades que se producen en su adquisición (pruebas como el TEDI-MATH, PRECUMAT, PDM, test de evolución matemática temprana de Utrecht). Entre estas aportaciones se encuentra el Test de Competencia Matemática Básica, TEMA-3 (Test of Early Mathematical Ability, GINSBURG; BAROODY, 2007). Sus particulares características de diseño lo sitúan como opción preferente para valorar la competencia aritmética temprana. A partir de la revisión de gran parte de los resultados de investigación reciente, plantea el desarrollo del pensamiento matemático infantil, de forma lúdica, ágil y sencilla, señalando la distinción entre conocimiento matemático informal y conocimiento matemático formal. En concreto, comienza con la evaluación del pensamiento matemático informal, aquel que se desarrolla tempranamente, sin mediar instrucción formal, como respuesta a las necesidades prácticas y las experiencias concretas de los niños en relación con el número y la cantidad en sus contextos cotidianos. Más adelante, se introduce la evaluación del conocimiento matemático formal, qué será desarrollado en el marco académico, implicando necesariamente el dominio de la numeración posicional y los algoritmos de cálculo. Se trata, por tanto, de una prueba que valora tanto aspectos informales (aprendidos en contextos variados, no necesariamente en el entorno escolar) como formales (ligados a los símbolos convencionales, y transmitidos en el medio escolar), diferenciando entre habilidades (como la resolución de operaciones de cálculo) y conceptos. Su aplicación individual permite atender aspectos claves con relación a cómo el alumno afronta las situaciones aritméticas que le son planteadas, aportando información y pistas relevantes para 
orientar la mejora de los aprendizajes y/o la recuperación de las dificultades detectadas.

Por ello, este trabajo se propone utilizar las potencialidades del test TEMA-3 para la evaluación de las habilidades matemáticas de alumnos de 3ํㅡㄹ Educación Infantil. En concreto, se pretenden los siguientes objetivos:

1) describir su competencia matemática media, en comparación con el grupo normativo;

2) analizar su perfil de ejecución medio, para descubrir las fortalezas y debilidades en su desarrollo;

3) detectar alumnos con rendimientos significativamente por encima o por debajo de la media de su curso de referencia, ilustrando cómo derivar orientaciones para la intervención que se adapte específicamente a sus necesidades individuales, con el objetivo de promover la optimización de su nivel de rendimiento, en función del perfil concreto mostrado;

4) analizar las relaciones entre las habilidades matemáticas y habilidades cognitivas generales, así como sus relaciones con el rendimiento en matemáticas.

\section{Método}

Participantes: la muestra, extraída de forma incidental, está compuesta por 55 alumnos escolarizados en un centro concertado del área Metropolitana de Madrid, constituyendo dos grupos naturales de aula de $3^{\text {o }}$ de EI. La edad cronológica estaba comprendida entre los valores 5.38 y 6.58 (con $X=5.88$ y s $=0.29$ ), siendo el $54.5 \%$ niños y el $45.5 \%$ niñas.

Instrumentos: se aplicaron las pruebas TEMA-3 (GINSBURG; BAROODY, 2007) y BADyG-1 (Batería de Aptitudes Diferenciales y Generales; YUSTE, 2001). Además se pidió a los profesores la calificación del nivel de rendimiento general de cada alumno en relación a la habilidad aritmética en una escala de 1 a 10 puntos. 
El test TEMA-3 consta, en total, de 72 elementos, presentados en orden de dificultad creciente, en función de la edad, distribuidos en varios aspectos que valoran tanto habilidades como conceptos de carácter informal y formal. Para cada alumno, además de las puntuaciones clásicas (puntuación directa, edad y curso equivalentes, índice de competencia matemática (ICM) y percentil), se obtiene un perfil de ejecución, en el que se reflejan sus fortalezas y debilidades, siendo su análisis un valioso punto de partida para esclarecer las dificultades específicas de un alumno concreto y articular la intervención.

El aspecto informal del pensamiento matemático se valora a través de 41 ítems, distribuidos en cuatro componentes: a) numeración, que valora el dominio de la secuencia numérica, a través de tareas que implican habilidades básicas y avanzadas, tanto de conteo como de enumeración; b) comparación de cantidades, que implica la habilidad de establecer distancias relativas entre los números; c) cálculo informal, donde se plantean sencillas situaciones de suma y resta con objetos concretos y después se valoran las habilidades de cálculo mental (no necesariamente automático), y, d) conceptos básicos, donde se considera el uso de la regla de cardinalidad, la comprensión de la constancia numérica, la aplicación de estrategias de conteo avanzadas y la comprensión básica del reparto de objetos. El aspecto formal se evalúa a través de 31 ítems, a su vez, también repartidos en cuatro componentes: a) convencionalismos de lecto-escritura de cantidades, b) dominio de hechos numéricos, donde se mide la capacidad de recuperar de forma automática el resultado de operaciones de sumar, restar y multiplicar, con cantidades de un dígito - las tablas -; c) cálculo formal, donde se valora tanto la seguridad como el procedimiento seguido, $\mathrm{y}, \mathrm{d}$ ) conceptos básicos relacionados con el sistema numérico decimal, evaluados a través de ítems que implican la comprensión del valor posicional y las equivalencias entre los distintos órdenes de magnitud.

La prueba BADyG (Batería de Aptitudes Diferenciales y Generales) consta de 138 ítems distribuidos en categorías referidas a las siguientes habilidades: Habilidad mental no verbal (HMNV), Conceptos cuantitativos numéricos $(\mathrm{CN})$, Razonamiento con figuras (RL), Información (INF), 
Rompecabezas (RPC), Vocabulario gráfico (VG), Percepción auditiva/Reproducción de palabras (PA) y Percepción y coordinación grafo-motriz (PCGM).

Las puntuaciones globales permiten obtener un índice general de madurez intelectual global (IG) que resulta de las puntuaciones directas de la inteligencia general verbal (IGV) y de la no verbal (IGnV). La inteligencia general verbal (IGV), entendida como capacidad intelectual verbal y capacidad para asimilar conceptos numéricos y verbales, se obtiene de las pruebas referidas a los conceptos numéricos (CN), a la información (INF) y al vocabulario gráfico (VG). La inteligencia general no verbal (IGnV) resulta de la suma de los subtests referidos a la habilidad mental no verbal (HMnV), al razonamiento prelógico (RL), la habilidad para resolver problemas de tipo figurativo, encontrando una característica común a una serie de dibujos (RPC).

Procedimiento: la aplicación de la prueba TEMA-3 se realizó de forma individual, a cada uno de los alumnos de las aula de $3^{\circ}$ de EI, en una sala acondicionada para ello y dentro del horario escolar. La aplicación del test BADyG se realizó colectivamente, en las aulas correspondientes. Las pruebas se aplicaron a lo largo de las tres primeras semanas del mes de mayo de 2009. Se recogieron las calificaciones de los alumnos a final de curso, en el mes de junio.

Con relación al análisis estadístico de los datos, para el contraste del rendimiento se aplicó la técnica del ANOVA simple para grupos independientes, tomando como variables dependientes tanto la puntuación total del TEMA-3, como las puntuaciones correspondientes a cada uno de sus componentes; se contrastaron las diferencias entre el total de la muestra (grupo B) y el grupo normativo (grupo A), y entre los dos grupos de aula (grupos B1 y B2). Ante los resultados obtenidos, y entendiendo que podría producirse un sesgo debido a realizar el contraste considerando el total de la muestra normativa (evaluada en dos períodos del curso escolar, en el primer trimestre y a final de curso), se procedió a su división, seleccionado los datos correspondientes sólo a los alumnos valorados en el período equivalente de exploración (es decir, aquellos valorados en el mes de mayo). El ANOVA fue repetido, entonces, considerando sólo esta submuestra normativa de referencia. 
El estudio del rendimiento particular en cada ítem se valoró a través de las diferencias de proporciones de acierto, aplicando "Chi Cuadrado".

También fueron realizados análisis de correlación para abordar las relaciones entre las habilidades cognitivas generales (BADyG), las específicas de matemáticas (TEMA-3) y el rendimiento de los alumnos.

La selección de casos que precisan mayor atención, por obtener resultados significativamente por debajo o por encima de sus iguales, se realizó en función de percentiles de rendimiento global y análisis cualitativo del perfil de ejecución.

\section{Resultados}

Para dar respuesta a los objetivos planteados, primero se presenta una visión general del nivel de rendimiento alcanzado por la muestra objeto de estudio (primero considerada conjuntamente y después cada aula por separado). El contraste con el grupo normativo permitirá conocer en qué medida los resultados obtenidos se corresponden con los esperables según su edad y la altura de curso en la que fueron tomados los datos; en segundo lugar, el análisis del perfil de ejecución medio del grupo-aula concretará los aspectos superados como grupo, así como aquellos que necesitan refuerzo, que serán los que, concretamente, conformarán los objetivos de las orientaciones específicas para ser específicamente atendidos en la intervención en el aula. Los datos específicos de ciertos alumnos permitirán ilustrar el análisis cualitativo del TEMA-3, y finalmente los estudios correlacionales indicarán el grado de relación entre las puntuaciones de la prueba de competencia matemática específica y las habilidades en general con el nivel de rendimiento académico de los alumnos.

En términos generales, el rendimiento de esta muestra de alumnos de $3^{\circ}$ de EI se puede caracterizar a través del estudio de los niveles individuales de logro. La Tabla 1 recoge la distribución de las frecuencias de los resultados obtenidos por los alumnos reflejados en el Índice de Competencia Matemática (ICM; media de 100 y una desviación típica de 
15) agrupados en función de las categorías de interpretación que ofrece el manual de la prueba (GINSBURG; BAROODY, 2007, p. 68). Se observa predominio de la categoría de rendimiento "medio" (110 $\geq \mathrm{ICM} \geq 90$; $45.45 \%$ ), seguido por las categorías de "inferior a la media" (89 $\geq \mathrm{ICM} \geq$ $80 ; 21.82 \%)$ y "superior a la media" (120 $\geq$ ICM $\geq 111$; $12.73 \%)$; el porcentaje restante se encuentra muy repartido en tres categorías: "superior" (130 $\geq \mathrm{ICM} \geq 121 ; 5.45 \%)$, "pobre" (79 $\geq \mathrm{ICM} \geq 70 ; 10.91 \%)$ y "muy pobre" (ICM < 70; 3.64\%). Un análisis detallado de la distribución por aulas permite advertir ligeras diferencias entre ellas, siendo el aula B2 la que cuenta con mayor porcentaje de alumnos por encima de la media, mientras que el aula B1 atiende más alumnos con niveles de ejecución más limitados.

Tabla 1 - Distribución del Índice de Competencia Matemática de los grupos-aula y del total de la muestra

\begin{tabular}{|c|c|c|c|c|c|c|}
\hline \multirow[b]{2}{*}{ Intervalo } & \multicolumn{2}{|c|}{ Aula B1 } & \multicolumn{2}{|c|}{ Aula B2 } & \multicolumn{2}{|c|}{ Total: grupo B } \\
\hline & $\mathbf{N}$ & $\%$ & $\mathbf{N}$ & $\%$ & $\mathbf{N}$ & $\%$ \\
\hline$>131$ & 2 & 7.14 & 1 & 3.70 & 3 & 5.45 \\
\hline $121-130$ & 0 & 0 & 0 & 0 & 0 & 0 \\
\hline $111-120$ & 1 & 3.57 & 6 & 22.22 & 7 & 12.73 \\
\hline $90-110$ & 14 & 50.00 & 11 & 40.74 & 25 & 45.45 \\
\hline $80-89$ & 5 & 17.86 & 7 & 25.93 & 12 & 21.82 \\
\hline $70-79$ & 5 & 17.86 & 1 & 3.70 & 6 & 10.91 \\
\hline$<70$ & 1 & 3.57 & 1 & 3.70 & 2 & 3.64 \\
\hline
\end{tabular}

Fuente: Datos de la investigación.

Con el propósito de valorar el rendimiento medio de los alumnos de la muestra en la prueba de competencia matemática básica, TEMA-3, se contrastará a través del análisis de varianza de un factor la existencia de 
diferencias significativas entre las medias, tanto de la puntuación total, como de los diferentes componentes de la prueba. La Tabla 2 muestra los datos descriptivos correspondientes a las medidas de las distintas variables en las dos muestras para cada uno de los componentes analizados, así como los valores de t encontrados y sus probabilidades asociadas. Puede observarse cómo ciertas variables alcanzan significación estadística.

Tabla 2 - Estadísticos de grupo y contraste t para la igualdad de medias

\begin{tabular}{|c|c|c|c|c|c|c|c|c|}
\hline & \multirow[b]{2}{*}{$\mathrm{Gr}$} & \multirow[b]{2}{*}{$\mathbf{N}$} & \multirow[b]{2}{*}{ Media } & \multirow[b]{2}{*}{$\sigma$} & \multirow[b]{2}{*}{ ET } & \multicolumn{3}{|c|}{$\begin{array}{l}\text { Prueba t para la } \\
\text { igualdad de medias }\end{array}$} \\
\hline & & & & & & $\mathbf{t}$ & gl & Sig (bilateral) \\
\hline \multirow[t]{2}{*}{ Total TEMA-3 } & A & 101,00 & 23,84 & 7,32 & 0,73 & $3,38 * *$ & 154,00 & 0,0009 \\
\hline & B & 55,00 & 27,93 & 7,04 & 0,95 & & & \\
\hline \multirow[t]{2}{*}{ Numeración } & A & 101,00 & 12,17 & 3,20 & 0,32 & $3,61^{* *}$ & 154,00 & 0,0004 \\
\hline & B & 55,00 & 14,15 & 3,38 & 0,46 & & & \\
\hline \multirow[t]{2}{*}{ Comparación } & A & 101,00 & 3,03 & 1,07 & 0,11 & $3,01 * *$ & 154,00 & 0,0030 \\
\hline & B & 55,00 & 3,56 & 1,03 & 0,14 & & & \\
\hline \multirow[t]{2}{*}{ Cálculo informal } & A & 101,00 & 2,78 & 1,25 & 0,12 & $5,11^{* *}$ & 124,39 & 0,0000 \\
\hline & B & 55,00 & 3,76 & 1,09 & 0,15 & & & \\
\hline \multirow{2}{*}{$\begin{array}{l}\text { Conceptos } \\
\text { informales }\end{array}$} & A & 101,00 & 2,16 & 0,50 & 0,05 & $0,46 * *$ & 154,00 & 0,6441 \\
\hline & B & 55,00 & 2,20 & 0,59 & 0,08 & & & \\
\hline \multirow[t]{2}{*}{ Convencionalismos } & A & 101,00 & 2,28 & 1,43 & 0,14 & $1,91 * *$ & 154,00 & 0,0583 \\
\hline & B & 55,00 & 2,73 & 1,37 & 0,18 & & & \\
\hline \multirow[t]{2}{*}{ Hechos numéricos } & A & 101,00 & 0,02 & 0,14 & 0,01 & $1,95 * *$ & 56,52 & 0,0562 \\
\hline & B & 55,00 & 0,20 & 0,68 & 0,09 & & & \\
\hline \multirow[t]{2}{*}{ Cálculo formal } & A & 101,00 & 0,01 & 0,10 & 0,01 & $0,70 * *$ & 62,12 & 0,4852 \\
\hline & B & 55,00 & 0,04 & 0,27 & 0,04 & & & \\
\hline \multirow{2}{*}{$\begin{array}{l}\text { Conceptos } \\
\text { formales }\end{array}$} & A & 101,00 & 0,96 & 0,20 & 0,02 & $0,93 * *$ & 154,00 & 0,3513 \\
\hline & B & 55,00 & 1,00 & 0,33 & 0,04 & & & \\
\hline
\end{tabular}

Fuente: Datos de la investigación.

Leyenda: grupo $\mathrm{A}=$ grupo normativo; grupo $\mathrm{B}=$ grupos de aula; ${ }^{*}=$ significación estadística de la diferencia de medias. 
Como ya ha sido advertido, ante la posibilidad de que este resultado se viera sesgado en razón del momento de exploración de los alumnos en relación al avance el curso escolar, se valoró la submuestra de referencia evaluada en el segundo período de curso, y se repitieron los ANOVA's. En este caso (ver tabla 3), desaparecen las diferencias entre ambas muestras.

Se puede afirmar, por tanto, tras este segundo análisis de los datos, que el nivel de rendimiento aritmético medio del grupo de alumnos

Tabla 3 - Estadísticos de grupo y contraste t para la igualdad de medias

\begin{tabular}{|c|c|c|c|c|c|c|c|c|}
\hline & \multirow[b]{2}{*}{ Gr } & \multirow[b]{2}{*}{$\mathbf{N}$} & \multirow[b]{2}{*}{ Media } & \multirow[b]{2}{*}{$\sigma$} & \multirow[b]{2}{*}{ ET } & \multicolumn{3}{|c|}{$\begin{array}{l}\text { Prueba t para la } \\
\text { igualdad de medias }\end{array}$} \\
\hline & & & & & & $\mathbf{t}$ & gl & Sig (bilateral) \\
\hline \multirow[t]{2}{*}{ Total TEMA-3 } & $A$ & 49,00 & 28,43 & 6,65 & 0,95 & $-0,37$ & 102,00 & 0,7106 \\
\hline & B & 55,00 & 27,93 & 7,04 & 0,95 & & & \\
\hline \multirow[t]{2}{*}{ Numeración } & $A$ & 49,00 & 13,92 & 3,09 & 0,44 & 0,36 & 102,00 & 0,7227 \\
\hline & B & 55,00 & 14,15 & 3,38 & 0,46 & & & \\
\hline \multirow[t]{2}{*}{ Comparación } & $A$ & 49,00 & 3,55 & 0,91 & 0,13 & 0,07 & 102,00 & 0,9478 \\
\hline & B & 55,00 & 3,56 & 1,03 & 0,14 & & & \\
\hline \multirow[t]{2}{*}{ Cálculo informal } & A & 49,00 & 3,59 & 0,84 & 0,12 & 0,89 & 102,00 & 0,3738 \\
\hline & B & 55,00 & 3,76 & 1,09 & 0,15 & & & \\
\hline \multirow{2}{*}{$\begin{array}{l}\text { Conceptos } \\
\text { informales }\end{array}$} & A & 49,00 & 2,39 & 0,57 & 0,08 & $-1,65$ & 102,00 & 0,1030 \\
\hline & B & 55,00 & 2,20 & 0,59 & 0,08 & & & \\
\hline \multirow[t]{2}{*}{ Convencionalismos } & A & 49,00 & 3,08 & 1,47 & 0,21 & $-1,27$ & 102,00 & 0,2057 \\
\hline & B & 55,00 & 2,73 & 1,37 & 0,18 & & & \\
\hline \multirow[t]{2}{*}{ Hechos numéricos } & A & 49,00 & 0,04 & 0,20 & 0,03 & 1,66 & 64,37 & 0,1012 \\
\hline & B & 55,00 & 0,20 & 0,68 & 0,09 & & & \\
\hline \multirow[t]{2}{*}{ Cálculo formal } & A & 49,00 & 0,02 & 0,14 & 0,02 & 0,37 & 102,00 & 0,7119 \\
\hline & B & 55,00 & 0,04 & 0,27 & 0,04 & & & \\
\hline \multirow{2}{*}{$\begin{array}{l}\text { Conceptos } \\
\text { formales }\end{array}$} & A & 49,00 & 1,00 & 0,00 & 0,00 & 0,00 & 102,00 & 1,0000 \\
\hline & B & 55,00 & 1,00 & 0,33 & 0,04 & & & \\
\hline
\end{tabular}

Fuente: Datos de la investigación.

Leyenda: grupo $\mathrm{A}$ = grupo normativo; grupo $\mathrm{B}=$ grupos de aula. 
considerado es similar al de su grupo normativo de referencia, analizando los resultados en el mismo período de evaluación (final de curso).

El estudio del perfil grupal de ejecución de los alumnos a través de los porcentajes de logro de cada uno de los elementos que integran los componentes del TEMA-3 (ver Tabla 4, página seguiente), junto al análisis de "Chi Cuadrado" con el fin de valorar posibles diferencias entre ellos, permite conocer si algún aspecto de la prueba obtiene rendimientos estadísticamente diferentes entre ambas muestras.

Sólo se obtienen diferencias significativas en dos ítems: lectura de números de dos cifras (entre 11 y 19; ítem 28), que muestra superioridad de la muestra normativa (grupo A), y la aplicación de técnicas de conteo más avanzadas (contar a partir del mayor; ítem 34), donde rinden mejor los alumnos del grupo analizado actualmente (grupo B). Se acerca a la significación, y a favor del grupo normativo (grupo A), el ítem que valora el dominio de la serie regresiva (ítem 25).

Analizando los porcentajes de ejecución podemos caracterizar el rendimiento de estos alumnos de $3^{\circ}$ de EI en función de sus fortalezas y debilidades (Tabla 4), y así, con relación a las habilidades informales de la competencia matemática manifiestan, como grupo, fortalezas en el uso de estrategias de conteo avanzadas aplicadas a la solución de planteamientos de situaciones verbales (saben las ventajas de contar a partir del mayor de los sumandos), mientras que sus debilidades afectan a limitaciones para recitar la serie inversa desde 10 y valorar cuál es el mayor entre dos dígitos consecutivos entre 5 y 10. En el desarrollo de las habilidades formales, como grupo, no se aprecian claras fortalezas, manifestándose en la evaluación actual ciertas dificultades en la lectura de cantidades entre 10 y 19.

Si bien es cierto que es importante conocer los niveles de rendimiento promedio de los grupos que conforman la comunidad de aprendizaje del aula, sin duda requiere especial atención el resultado individualizado de cada alumno. A través de los perfiles individuales pueden detectarse aquellos alumnos cuyo rendimiento haya sido especialmente inferior o superior a la media de su grupo de referencia. 
Tabla 4 - Porcentaje de logro en los ítems de la prueba TEMA-3. Valor $x^{2}$

(Continua)

\begin{tabular}{|c|c|c|c|c|c|c|c|}
\hline Pensamiento informal & $\begin{array}{c}\text { Gr A } \\
\%\end{array}$ & $\begin{array}{c}\text { Gr B } \\
\%\end{array}$ & $x^{2}$ & Pensamiento formal & $\begin{array}{c}\text { Gr A } \\
\%\end{array}$ & $\begin{array}{c}\text { Gr B } \\
\%\end{array}$ & $\chi^{2}$ \\
\hline \multicolumn{4}{|c|}{ Conteo: secuencia básica } & \multicolumn{4}{|c|}{ Convencionalismos } \\
\hline 2 - Patrón de dedos & 100 & 100 & & 14 - Lectura de dígitos & 100 & 100 & \\
\hline 3 - Numeración intuitiva & 100 & 100 & & 18 - Escritura de dígitos & 94 & 84 & $2,663^{*}$ \\
\hline 4 - Contar de 1 en 1 (5) & 100 & 100 & & 28 - Lectura de números & 57 & 36 & $4,502 *$ \\
\hline 5 - Produc. no verbal (4) & 100 & 100 & & 30 - Lectura de números & 20 & 24 & $0,157^{*}$ \\
\hline 9 - Contar de 1 en 1 (10) & 100 & 100 & & 31 - Escrit. de números & 24 & 29 & $0,279 *$ \\
\hline 10 - Mostrar dedos (5) & 100 & 100 & & 42 - Lectura de números & 8 & 2 & $2,280 *$ \\
\hline 20 - Sec. numérica (21) & 78 & 82 & 0,293 & 43 - Escrit. de números & 4 & 0 & $2,289 *$ \\
\hline 29 - Sec. numérica (42) & 39 & 44 & 0,252 & 55 - Lectura de números & 0 & 0 & \\
\hline
\end{tabular}

Conteo: enumeración

Hechos numéricos

\begin{tabular}{|c|c|c|c|c|c|c|c|}
\hline 6 - Enumeración (5) & 100 & 100 & & 36 - Resta $(\mathrm{N}-\mathrm{N} ; \mathrm{N}-1)$ & 4 & 9 & 1,036 \\
\hline 12 - Formar conjuntos & 100 & 100 & & 47 - Suma $(n<5)$ & 0 & 4 & 1,817 \\
\hline 22 - Enumeración (10) & 86 & 91 & 0,685 & 48 - Multip. $(\mathrm{N} \times 1 ; \mathrm{N} \times 0)$ & 0 & 0 & \\
\hline 27 - Formar conjuntos & 57 & 55 & 0,071 & $50-\operatorname{Resta}(M-N=N)$ & 0 & 0 & \\
\hline 40 - Enumeración (20) & 14 & 22 & 0,985 & $51-S \cdot(a+b=10 ; a+a<10)$ & 0 & 2 & 0,900 \\
\hline \multicolumn{4}{|c|}{ Conteo: sec. avanzada } & 52 - Suma (dobles) & 0 & 0 & \\
\hline 13 - Serie partida (9) & 94 & 93 & 0,055 & 61 - Resta $(10-N)$ & 0 & 0 & \\
\hline 21 - Serie partida (40) & 69 & 75 & 0,343 & 67 - Suma $n$ os grandes $^{-}$ & 0 & 2 & 0,900 \\
\hline 25 - Serie regresiva (10) & 80 & 45 & 3,214 & 68 - Multip. $(\mathrm{N} \times 2)$ & 0 & 0 & \\
\hline 32 - Serie partida (50) & 20 & 33 & 1,999 & \multicolumn{4}{|c|}{ Cálculo } \\
\hline 33 - Contar de 10 en 10 & 14 & 13 & 0,054 & 44 - Suma sin llevadas & 2 & & 1,133 \\
\hline 37 - Serie regresiva (20) & 16 & 15 & 0,063 & 49 - Alineación cuentas & & & \\
\hline 38 - Serie partida (99) & 12 & 13 & 0,006 & 54 - Sum. y rest. de 10 & 0 & 2 & 0,900 \\
\hline 41 - Sec. de $10-10$ (100) & 6 & 7 & 0,055 & 57 - Exactitud de suma & 0 & 0 & \\
\hline
\end{tabular}


Tabla 4 - Porcentaje de logro en los ítems de la prueba TEMA-3. Valor $x^{2}$

(Conclusión)

\begin{tabular}{|c|c|c|c|c|c|c|c|}
\hline Pensamiento informal & $\begin{array}{c}\text { Gr A } \\
\%\end{array}$ & $\begin{array}{c}\text { Gr B } \\
\%\end{array}$ & $\chi^{2}$ & Pensamiento formal & $\begin{array}{c}\mathrm{Gr} A \\
\%\end{array}$ & $\begin{array}{c}\text { Gr B } \\
\%\end{array}$ & $\chi^{2}$ \\
\hline \multicolumn{4}{|c|}{ Conteo: sec. avanzada } & \multicolumn{4}{|c|}{ Cálculo } \\
\hline 45 - Sec partida $(>100)$ & 6 & 5 & 0,021 & 58 - Proced. de suma & 0 & 0 & \\
\hline 66 - Contar de $\mathrm{n}$ en $\mathrm{n}$. & 0 & 0 & & 59 - Suma $(n \times 10)$ & 0 & 2 & 0,900 \\
\hline \multicolumn{4}{|c|}{ Magnitud relativa } & 63 - Resta $(n \times 10)$ & 0 & 0 & \\
\hline 1 - Percepción de más & 100 & 100 & & 69 - Exactitud de resta & 0 & 0 & \\
\hline 16 - Concepto de más & 94 & 98 & 1,298 & 70 - Proced.de resta & 0 & 0 & \\
\hline 17 - Concepto de más & 84 & 76 & 0,859 & \multicolumn{4}{|c|}{ Conceptos formales } \\
\hline 26 - Línea numérica (10) & 53 & 62 & 0,814 & 15 - Represent. escrita & 100 & 96 & 1,818 \\
\hline 35 - Línea numérica (100) & 24 & 24 & 0,010 & 53 - Dec. en 1 centena & 0 & 2 & 0,900 \\
\hline 60 - Línea numérica (+100) & 0 & 0 & & 56 - Cent. en 1 millar & 0 & 0 & \\
\hline \multicolumn{4}{|c|}{ Cálculo mental } & 64 - Valor posicional & 0 & 0 & \\
\hline 8 - Suma y resta no verbal & 100 & 100 & & 71 - Conmutatividad & 0 & 0 & \\
\hline 19 - Sumar objetos & 82 & 87 & 0,633 & & & & \\
\hline 23 - Sumar objetos (mod.) & 90 & 89 & 0,014 & & & & \\
\hline 24 - Suma mental & 84 & 78 & & & & & \\
\hline 34 - Contar desde mayor & 4 & 20 & 0,503 & & & & \\
\hline 62 - Suma mental & 0 & 2 & 0,900 & & & & \\
\hline 65 - Resta mental & 0 & 0 & & & & & \\
\hline 72 - Resta mental & 0 & 0 & & & & & \\
\hline \multicolumn{4}{|c|}{ Conceptos informales } & & & & \\
\hline 7 - Regla de cardinalidad & 100 & 100 & & & & & \\
\hline 11 - Constancia numérica & 100 & 98 & 0,900 & & & & \\
\hline 39 - Reparto equivalente & 27 & 18 & 0,737 & & & & \\
\hline 46 - Relac. partes-todo & 12 & 7 & 1,049 & & & & \\
\hline
\end{tabular}

Fuente: Datos de la investigación.

Leyenda: grupo $\mathrm{A}$ = grupo normativo; grupo $\mathrm{B}$ = grupo de aula.

Rev. Diálogo Educ., Curitiba, v. 11, n. 32, p. 83-105, jan./abr. 2011 
La Tabla 5 presenta los datos de cada alumno, en cada grupo de aula, resaltando los que deben ser especialmente considerados (se han señalado los que obtienen percentiles inferiores a 16 o superiores a 84 , ya que se alejan una desviación típica de la media normativa).

Tabla 5 - Resultados individuales en el TEMA-3

(Continua)

\begin{tabular}{rrrrrrrrrrrrrrrr}
\hline Caso & Pd & EC & INUM & ICO & ICA & ICP & FCO & FNU & FCA & FCP & NT & ICM & EE & PC \\
\hline 1 & 36 & 5,99 & 16 & 5 & 5 & 3 & 5 & 0 & 0 & 1 & 9,0 & 110 & 6,17 & 75 \\
2 & 32 & 5,79 & 16 & 3 & 5 & 3 & 4 & 0 & 0 & 1 & 8,0 & 102 & 5,92 & 57 \\
3 & 24 & 5,80 & 12 & 2 & 4 & 2 & 1 & 0 & 0 & 1 & 7,0 & 87 & 5,33 & 20 \\
4 & 27 & 5,82 & 15 & 3 & 4 & 2 & 2 & 0 & 0 & 1 & 5,0 & 93 & 5,58 & 34 \\
$\mathbf{5}$ & $\mathbf{3 2}$ & $\mathbf{5 , 5 8}$ & $\mathbf{1 4}$ & $\mathbf{4}$ & $\mathbf{4}$ & $\mathbf{3}$ & $\mathbf{5}$ & $\mathbf{1}$ & $\mathbf{0}$ & $\mathbf{1}$ & $\mathbf{9 , 0}$ & $\mathbf{1 1 7}$ & $\mathbf{5 , 9 2}$ & $\mathbf{8 8}$ \\
$\mathbf{6}$ & $\mathbf{2 1}$ & $\mathbf{6 , 5 8}$ & $\mathbf{1 1}$ & $\mathbf{3}$ & $\mathbf{3}$ & $\mathbf{2}$ & $\mathbf{1}$ & $\mathbf{0}$ & $\mathbf{0}$ & $\mathbf{1}$ & $\mathbf{2 , 0}$ & $\mathbf{6 4}$ & $\mathbf{5 , 0 8}$ & $<\mathbf{1}$ \\
$\mathbf{7}$ & 30 & 5,53 & 17 & 5 & 4 & 2 & 1 & 0 & 0 & 1 & 7,0 & 112 & 5,75 & 78 \\
8 & 32 & 6,14 & 16 & 4 & 5 & 2 & 3 & 1 & 0 & 1 & 6,0 & 102 & 5,92 & 57 \\
9 & 22 & 5,66 & 11 & 2 & 4 & 2 & 2 & 0 & 0 & 1 & 6,0 & 91 & 5,17 & 28 \\
10 & 25 & 5,78 & 12 & 3 & 4 & 2 & 3 & 0 & 0 & 1 & 5,0 & 88 & 5,42 & 22 \\
11 & 21 & 5,45 & 10 & 4 & 2 & 2 & 2 & 2 & 0 & 1 & 6,0 & 98 & 5,08 & 48 \\
12 & 24 & 5,71 & 11 & 4 & 4 & 2 & 2 & 0 & 0 & 1 & 6,0 & 87 & 5,33 & 20 \\
13 & 26 & 5,94 & 14 & 4 & 2 & 2 & 4 & 0 & 0 & 1 & 6,0 & 91 & 5,50 & 28 \\
14 & 28 & 6,31 & 14 & 4 & 4 & 2 & 3 & 0 & 0 & 1 & 5,0 & 87 & 5,67 & 20 \\
15 & 35 & 5,74 & 20 & 4 & 5 & 2 & 3 & 0 & 0 & 1 & 9,0 & 108 & 6,08 & 72 \\
16 & 25 & 5,81 & 12 & 3 & 4 & 2 & 1 & 0 & 0 & 1 & 6,0 & 89 & 5,42 & 23 \\
17 & 37 & 5,97 & 20 & 5 & 4 & 3 & 5 & 0 & 0 & 1 & 9,0 & 112 & 6,25 & 78 \\
18 & 28 & 6,16 & 13 & 4 & 4 & 2 & 3 & 0 & 0 & 1 & 6,0 & 94 & 5,67 & 37 \\
$\mathbf{1 9}$ & $\mathbf{1 9}$ & $\mathbf{5 , 8 6}$ & $\mathbf{1 1}$ & $\mathbf{2}$ & $\mathbf{1}$ & $\mathbf{1}$ & $\mathbf{2}$ & $\mathbf{0}$ & $\mathbf{0}$ & $\mathbf{1}$ & $\mathbf{2 , 0}$ & $\mathbf{7 7}$ & $\mathbf{4 , 8 3}$ & $\mathbf{4}$ \\
20 & 21 & 5,81 & 10 & 3 & 4 & 2 & 1 & 0 & 0 & 1 & 4,0 & 88 & 5,08 & 22 \\
21 & 28 & 6,34 & 15 & 3 & 4 & 2 & 3 & 0 & 0 & 1 & 9,0 & 87 & 5,67 & 20 \\
22 & 38 & 6,09 & 18 & 5 & 5 & 3 & 5 & 0 & 0 & 1 & 7,0 & 114 & 6,25 & 83 \\
$\mathbf{2 3}$ & $\mathbf{4 4}$ & $\mathbf{5 , 7 0}$ & $\mathbf{2 1}$ & $\mathbf{5}$ & $\mathbf{5}$ & $\mathbf{4}$ & $\mathbf{5}$ & $\mathbf{2}$ & $\mathbf{0}$ & $\mathbf{1}$ & $\mathbf{9 , 0}$ & $\mathbf{1 4 8}$ & $\mathbf{6 , 6 7}$ & $\mathbf{9 9 , 8}$ \\
24 & 22 & 5,50 & 11 & 3 & 3 & 2 & 2 & 0 & 0 & 1 & 4,0 & 91 & 5,17 & 28 \\
$\mathbf{2 5}$ & $\mathbf{2 7}$ & $\mathbf{5 , 4 3}$ & $\mathbf{1 4}$ & $\mathbf{4}$ & $\mathbf{4}$ & $\mathbf{2}$ & $\mathbf{2}$ & $\mathbf{0}$ & $\mathbf{0}$ & $\mathbf{1}$ & $\mathbf{6 , 0}$ & $\mathbf{1 1 8}$ & $\mathbf{5 , 5 8}$ & $\mathbf{8 9}$ \\
26 & 38 & 6,13 & 20 & 5 & 4 & 3 & 5 & 0 & 0 & 1 & 9,0 & 114 & 6,25 & 83 \\
& & & & & & & & & & & & &
\end{tabular}


Tabla 5 - Resultados individuales en el TEMA-3

(Conclusión)

\begin{tabular}{|c|c|c|c|c|c|c|c|c|c|c|c|c|c|c|}
\hline Caso & $\mathbf{P d}$ & EC & INUM & ICO & ICA & ICP & FCO & FNU & FCA & FCP & NT & ICM & EE & PC \\
\hline 27 & 33 & 6,14 & 16 & 5 & 5 & 2 & 4 & 0 & 0 & 1 & 9,0 & 104 & 6,00 & 62 \\
\hline 28 & 20 & 5,93 & 12 & 2 & 2 & 2 & 1 & 0 & 0 & 1 & 4,5 & 79 & 5,00 & 6 \\
\hline 29 & 28 & 5,84 & 13 & 2 & 4 & 2 & 1 & 0 & 0 & 1 & 7,0 & 95 & 5,67 & 40 \\
\hline 30 & 26 & 6,14 & 12 & 4 & 4 & 2 & 2 & 0 & 0 & 1 & 8,0 & 90 & 5,50 & 25 \\
\hline 31 & 27 & 5,64 & 14 & 3 & 3 & 2 & 4 & 0 & 0 & 1 & 6,0 & 104 & 5,58 & 62 \\
\hline 32 & 28 & 5,83 & 14 & 5 & 4 & 2 & 2 & 0 & 0 & 1 & 6,0 & 95 & 5,67 & 40 \\
\hline 33 & 13 & 6,07 & 7 & 1 & 1 & 2 & 2 & 0 & 0 & 0 & 2,0 & 64 & 4,17 & $<1$ \\
\hline 34 & 26 & 6,13 & 14 & 4 & 3 & 2 & 2 & 0 & 0 & 1 & 7,0 & 84 & 5,50 & 13 \\
\hline 35 & 26 & 6,34 & 14 & 3 & 4 & 2 & 2 & 0 & 0 & 1 & 6,5 & 84 & 5,50 & 13 \\
\hline 36 & 29 & 5,68 & 16 & 4 & 4 & 2 & 3 & 0 & 0 & 1 & 7,5 & 96 & 5,67 & 42 \\
\hline 37 & 20 & 6,16 & 10 & 2 & 3 & 1 & 2 & 0 & 0 & 1 & 8,0 & 78 & 5,00 & 7 \\
\hline 38 & 35 & 5,82 & 18 & 4 & 5 & 2 & 5 & 0 & 0 & 1 & 9,0 & 108 & 6,08 & 72 \\
\hline 39 & 50 & 5,42 & 22 & 5 & 6 & 4 & 5 & 4 & 2 & 3 & 10,0 & $>150$ & 7,08 & 99,9 \\
\hline 40 & 26 & 6,36 & 13 & 3 & 4 & 2 & 2 & 0 & 0 & 1 & 7,0 & 84 & 5,50 & 13 \\
\hline 41 & 27 & 5,78 & 14 & 4 & 4 & 2 & 2 & 0 & 0 & 1 & 6,0 & 93 & 5,58 & 34 \\
\hline 42 & 19 & 6,11 & 9 & 2 & 3 & 2 & 2 & 0 & 0 & 1 & 6,0 & 76 & 4,83 & 4 \\
\hline 43 & 26 & 6,27 & 11 & 4 & 4 & 2 & 2 & 0 & 0 & 1 & 6,0 & 84 & 5,50 & 13 \\
\hline 44 & 26 & 5,79 & 15 & 3 & 2 & 2 & 3 & 0 & 0 & 1 & 6,0 & 91 & 5,50 & 28 \\
\hline 45 & 20 & 5,43 & 13 & 3 & 2 & 2 & 1 & 0 & 0 & 0 & 5,5 & 95 & 5,00 & 40 \\
\hline 46 & 41 & 6,25 & 20 & 5 & 5 & 4 & 4 & 1 & 0 & 1 & 9,0 & 107 & 6,50 & 69 \\
\hline 47 & 25 & 5,46 & 13 & 3 & 4 & 2 & 2 & 0 & 0 & 1 & 6,5 & 112 & 5,42 & 78 \\
\hline 48 & 25 & 5,38 & 11 & 4 & 4 & 2 & 2 & 0 & 0 & 1 & 6,5 & 78 & 5,42 & 78 \\
\hline 49 & 29 & 5,81 & 16 & 4 & 4 & 2 & 2 & 0 & 0 & 1 & 8,5 & 97 & 5,67 & 45 \\
\hline 50 & 33 & 5,81 & 17 & 5 & 4 & 2 & 4 & 0 & 0 & 1 & 7,5 & 104 & 6,00 & 62 \\
\hline 51 & 25 & 6,01 & 13 & 3 & 4 & 2 & 2 & 0 & 0 & 1 & 8,5 & 88 & 5,42 & 22 \\
\hline 52 & 15 & 5,47 & 8 & 2 & 1 & 2 & 1 & 0 & 0 & 1 & 3,0 & 78 & 4,42 & 5 \\
\hline 53 & 27 & 6,08 & 14 & 4 & 4 & 2 & 2 & 0 & 0 & 1 & 8,0 & 92 & 5,58 & 31 \\
\hline 54 & 29 & 6,19 & 15 & 4 & 4 & 2 & 3 & 0 & 0 & 1 & 7,0 & 96 & 5,67 & 42 \\
\hline 55 & 40 & 5,55 & 20 & 3 & 5 & 3 & 6 & 0 & 0 & 1 & 9,0 & 138 & 6,42 & 99,4 \\
\hline
\end{tabular}

Fuente: Datos de la investigación. 
Atendiendo inicialmente la necesidad que se manifiesta en el perfil de los alumnos que obtienen puntuaciones claramente por debajo de su nivel de referencia, los datos reflejados permiten establecer tres grupos claramente diferenciados. El primero incluiría cuatro alumnos (casos 33, 34, 40, 43) cuya ejecución alcanza los 5 años y 6 meses, y aunque se encuentran en un percentil bajo es razonable esperar que con un pequeño esfuerzo, reforzando las actividades de secuencia numéricas de su propio nivel, logren niveles de rendimiento semejantes al grupo de iguales. Un segundo grupo estaría formado por cinco alumnos cuyo desarrollo matemático alcanza una edad entre los 4 años y 6 meses y los 5 años y 0 meses (casos $6,19,28,37,42$ ). Sería conveniente reforzar específicamente las habilidades de secuencia numérica, insistiendo en la adquisición de numerales para ampliar su dominio, planteando específicamente tareas de secuencia avanzada (secuencia partida y secuencia inversa) y de numeración, dada su falta de dominio en estos aspectos. Dos alumnos obtienen edades equivalentes por debajo de 4 años y 6 meses (casos 33, 52): sus dificultades recomiendan realizar adaptaciones significativas para poder ajustar las actividades de enseñanza a sus actividades particulares. Sería conveniente, además de plantear actividades de refuerzo de secuencia numérica, abordar las tareas de comparación de cantidades. Para facilitar la adquisición y asimilación de estas habilidades se recomienda disponer de materiales concretos que puedan utilizar en sus recuentos, así como una línea numérica visible en sus mesas de trabajo (hasta 20 por ejemplo).

Es importante atender el grupo de alumnos cuyo desarrollo matemático alcanza niveles muy superiores a la media del grupo de referencia. Destacan tres alumnos que superan el percentil 99 (casos 23, 39, 55). Dado su potencial debería valorarse la necesidad de ofrecer actividades de ampliación que abordaran la memorización de hechos numéricos y la resolución de cálculos formales, apoyándose en su dominio de la secuencia numérica y del código numérico convencional. A lo largo del proceso de intervención es indispensable realizar un seguimiento cercano de la evolución de estos alumnos que permita realizar los ajustes oportunos. 
Con fines descriptivos se calcularon y analizaron las correlaciones entre la prueba TEMA-3, específica de desarrollo matemático, y el BADYG, que valora de forma más amplia el desarrollo general; a su vez, se valoró la relación de ambas con el rendimiento matemático de los alumnos, a partir de las valoraciones aportadas por los profesores-tutores del aula, como se refleja en la Tabla 6 (página seguiente). La correlación más alta se encontró en los resultados en el TEMA-3 y la calificación en matemáticas $(r=.769, p<0.01)$. Destacan también las correlaciones con el subtest de conceptos numéricos del BADyG (con el TEMA-3, $\mathrm{r}=.547$, $\mathrm{p}<0.01$; con el rendimiento matemático, $r=.650, \mathrm{p}<0.01)$.

Tabla 6 - Correlaciones: TEMA-3, BADyG y rendimiento en matemáticas, $\mathrm{N}=55$

\begin{tabular}{lcc}
\hline & TEMA-3 & Nota matemáticas \\
\hline TEMA-3 & 1 &, $769^{(* *)}$ \\
NOTAMAT &, $769^{(*)}$ & 1 \\
IG &, $504^{(*)}$ &, $652^{(*)}$ \\
IGV &, $544^{(*)}$ &, $650^{(* *)}$ \\
IGnV &, $365^{(* *)}$ &, $521^{(* *)}$ \\
HMNV & 0,176 &, $292^{(*)}$ \\
CN &, $547^{(* *)}$ &, $650^{(* *)}$ \\
RL & 0,095 & 0,223 \\
INF &, $323^{(*)}$ &, $512^{(* *)}$ \\
RPC &, $394^{(* *)}$ &, $534^{(* *)}$ \\
VG &, $486^{(* *)}$ &, $468^{(* *)}$ \\
PCGM &, $455^{(* *)}$ &, $539^{(* *)}$ \\
\hline
\end{tabular}

Fuente: Datos de la investigación.

Leyenda: ${ }^{* *}=$ indica significación estadística del coeficiente de correlación al 0.01 ; $^{*}=$ indica significación estadística del coeficiente de correlación al 0.05 . 


\section{Discusión}

Los resultados obtenidos en el análisis del rendimiento aritmético temprano de estos dos grupos de aula de 3ํㅡㄹ de Educación Infantil permiten afirmar que, como grupo, obtienen promedios semejantes a sus iguales.

Además, el análisis de los porcentajes de logro en cada uno de los elementos del test revela las fortalezas y las debilidades de los alumnos (considerados como grupo) facilitando la adaptación de recursos didácticos y la planificación de actividades orientadas a la mejora del rendimiento en aquellos aspectos que lo necesitan. Y así, puede señalarse la conveniencia de reforzar, atendiendo a los aspectos informales del desarrollo matemático temprano, las habilidades avanzadas de secuencia numérica que implican el aprendizaje, uso y aplicación de la serie regresiva, ya que posteriormente resultará necesaria y de gran utilidad para resolver cálculos de resta. También necesitan mejorar sus habilidades de comparación de dígitos consecutivos. En relación al componente formal del desarrollo del pensamiento matemático se detecta la necesidad de insistir en actividades de lecto-escritura de cantidades dado su papel central en el avance de la competencia matemática básica.

No cabe duda de que conocer el nivel de rendimiento objetivo del grupo, así como reflexionar y profundizar en aspectos relacionados con las características concretas de sus dificultades, ha de repercutir favorablemente en la calidad de la atención pedagógica que el profesor ofrece a sus alumnos, considerados tanto individual como grupalmente, promoviendo la continua búsqueda de estrategias para mejorar su práctica docente (KILDAY; KINZEY, 2009).

En esta línea, los estudios de Baroody y Benson (2001) y Huber y Lenhoff (2006) aportan propuestas de intervención que subrayan la necesidad de crear "oportunidades" para aprender, ofreciendo a los alumnos problemas para que exploren, diseñen sus propias estrategias y trabajen con materiales concretos mientras van pensando. Un aula con un ambiente rico en "matemáticas" fortalecerá y promoverá la mejor base para lograr su aprendizaje de forma significativa. La calidad de la instrucción - 
referida a contenidos geométricos - tiene lugar, según Brown (2009), en ambientes que comparten tres características: utilizar un lenguaje matemático rico y preciso, animar el pensamiento de los niños y nutrir sus ideas y exploraciones.

Desde hace un tiempo, Baroody y Coslick (1998) proponen el "enfoque de investigación" como la mejor iniciativa para mejorar todos los aspectos del "poder" matemático. Su característica principal es que las tareas de aprendizaje se apoyan en proyectos, significados y preguntas. Es decir, se parte de una tarea interesante y a la vez retadora, que merece la pena, y que crea una necesidad real de aprender o de trabajar con las matemáticas. Sin duda, las tareas surgen de formas diversas: un problema atractivo, un proyecto, preguntas realizadas por los propios alumnos o se derivan, directamente, de situaciones cotidianas. Es más probable que la enseñanza desde este enfoque, por proyectos, desarrolle una disposición positiva, animando a los niños a utilizar y construir sobre la base de su conocimiento, esto es, aprovechar su conocimiento cotidiano, informal, integrándolo con lo enseñado en la escuela, el conocimiento formal de las matemáticas.

Con el mismo propósito de reforzar la competencia temprana de los alumnos, Ginsburg y Seo (2000) llaman la atención sobre el papel que puede jugar la lectura. A partir del interés de los niños, y en general su gusto por escuchar historias, animan a los profesores de estas etapas a realizar un esfuerzo por resaltar los contenidos matemáticos que se dan en ellas.

En conclusión, parece demostrado que la prueba TEMA-3 establece el nivel de competencia matemática básica y ofrece el perfil de ejecución que caracteriza el desempeño de los alumnos en estas habilidades, a partir de datos estandarizados y actualizados en población española. Una versión anterior (TEMA-2; NÚÑEZ; LOZANO, 2005) había mostrado su potencial en la evaluación del progreso aritmético de alumnos con discapacidad cognitiva. Este estudio ha comprobado su utilidad para detectar dificultades tempranas en el desarrollo de las habilidades numéricas en alumnos de 3ํㅡㄹ de EI. Estudios posteriores deberían valorar la eficacia de las orientaciones ofrecidas, realizando un seguimiento del progreso de los alumnos. 
Finalmente, sería conveniente dirigir esfuerzos hacia la elaboración y validación de nuevas pruebas de evaluación que cubrieran edades de desarrollo más tardío (al menos hasta el final de la Educación Básica Obligatoria).

\section{Referencias}

BAROODY, A. J. El pensamiento matemático de los niños: un marco evolutivo para maestros de preescolar, ciclo inicial y educación especial. Madrid: Aprendizaje Visor - MEC, 1988.

BAROODY, A. J. Does mathematics instruction for 3 to 5 -year olds really makes sense? Young Children, v. 55, n. 4, p. 61-67, 2000.

BAROODY, A. J. Number and operations: key transitions in the numerical and arithmetic development of typical and special children between the age of 2 and 6 years. Disponible en: <http://www.gse.buffalo.edu/org/conference/conferencepaper/Baroody.html >. Acceso en: 1 febr. 2001.

BAROODY, A. J.; BENSON, A. P. Early number instruction. Teaching Children Mathematics, v. 8, n. 3, p. 154-158, 2001.

BAROODY, A. J.; COSLICK, R. T. Fostering children's mathematical power: an investigative approach to k-8 Mathematics Instruction. Mahwah, NJ: LEA, 1998.

BAROODY, A. J.; DOWKER, A. The development of arithmetics concepts and skills: Constructing adaptative expertise. Mahwah, NJ: LEA, 2003.

BRISSIAUD, R. El aprendizaje del cálculo: más allá de Piaget y de la teoría de los conjuntos. Madrid: Antonio Machado, 1993.

BROWN, C. S. More than just number. Teaching Children Mathematics, v. 15, n. 8, p. 474-479, 2009.

DOWKER, A. Individual differences in numerical abilities in preschoolers. Developmental Science, v. 11, n. 5, p. 650-654, 2008. 
FUSON, K. C.; RICHARDS, J.; BRIARS, D. J. The acquisition and elaboration of the number word sequence. In: BRAINED, C. (Ed). Children's logical and mathematical cognition. New York: Springer-Verlarg, 1982. p. 33-91.

GIL, M. D.; VICENT, C. Análisis comparativo de la eficacia de un programa lúdico-narrativo para la enseñanza de las matemáticas en educación infantil. Psicothema, v. 21, n. 1, p. 70-75, 2009.

GINSBURG, H. P.; BAROODY, A. J. TEMA-3. Test de competencia matemática básica. Madrid: TEA Ediciones. Adaptación española: NÚÑEZ, M.C.; LOZANO, I, 2007.

GINSBURG, H. P.; SEO, K. Preschoolers' mathematical reading. Teaching Children Mathematics, v. 7, n. 4, p. 226-229, 2000.

HUBER, L. L.; LENHOFF, R. S. Mathematical concepts come alive in Pre-K and kindergarten classrooms. Teaching Children Mathematics, v. 13, n. 4, p. 226231, 2006.

KAMII, C. K. El niño reinventa la aritmética: implicaciones de la teoría de Piaget. Madrid: Aprendizaje Visor, 1985.

KAMII, C. K. El niño reinventa la aritmética II. Madrid: Aprendizaje Visor, 1992. KAMII, C. K. El niño reinventa la aritmética III: implicaciones de la teoría de Piaget. Madrid: Aprendizaje Visor, 1995.

KILDAY, C. R.; KINZIE, M. B. An analysis of instruments that measure the quality of mathematics teaching in early childhood. Early Childhood Education Journal, v. 36, n. 4, p. 365-372, 2009.

NCTM. Principles and standards for school mathematics. Reston: NCTM, 2000. NUNES, T.; BRYANT, P. Children doing mathematics. Oxford: Blackwell Publishers, 1996.

NÚÑEZ, M. C.; LOZANO, I. Evolución del rendimiento matemático temprano en una muestra de alumnos con discapacidad intelectual, mediante la prueba TEMA-2. Infancia y Aprendizaje, v. 28, n. 1, p. 39-50, 2005. 
RESNICK, L. B. A developmental theory of number understanding. In: GINSBURG, H. P. (Ed). The development of mathematical thinking. New York: Academic Press, 1983. p. 153-196.

YUSTE, C. Manual Técnico. BADyG. Madrid: CEPE, S.L., 2001.

Recibido: 15/08/2010

Recebido: 15/08/2010

Aprobado: 01/10/2010

Aprovado: 01/10/2010 